\title{
Dimension Reduction in fMRI Images based on Metaheuristic Algorithm to Diagnose Autism
}

\author{
Farzaneh Sadeghian, Hadiseh Hasani", Marzieh Jafari
}

Department of Geodesy and Surveying Engineering, Tafresh University, Tafresh, Iran

Article Info:

Received: 26 Jan 2021

Revised: 1 June 2021

Accepted: 3 June 2021

\section{A BSTRACT}

Introduction: Autism Spectrum Disorder (ASD) is a mental disorder and affects a person's linguistic skills and social interactions. With the production of Functional Magnetic Resonance Imaging (fMRI) and the development of their processing tools, the use of these images in identifying and evaluating the brain function of autistic people received a lot of attention. However, in this approach using the functional connectivity matrices leads to the creation of feature space with very high dimensions. Some of these features are dependent, unnecessary and additional, which reduces the quality of detection and increases the number of calculations. Therefore, regarding the large dimensions of the search space, the Particle Swarm Optimization (PSO) algorithm has been used as one of the powerful meta-heuristic search tools in selecting the optimal features. Materials and Methods: To evaluate the capability of the proposed method, the principal component analysis (PCA) algorithm is used as a standard dimension reduction method. In this study, the Support Vector Machines (SVM) classifier was used to detect autistic and healthy persons on the ABIDE database data. Feature space has been generated based on a functional connectivity matrix which has 6670 dimensions. Results: SVM accuracy in high-dimensional specialty space is $56 \%$. The proposed method based on PSO eliminates 3442 redundant features and increases classification accuracy up to $62.19 \%$ that performs better than PCA. The findings show that this meta-heuristic algorithm by removing almost half of the features results in a $6 \%$ increase in classification precision. Conclusion: The results indicate the ability of SVM in comparison with the Random Forest and K-Nearest Neighbor (KNN). PSO algorithm was used for dimension reduction of the input data space.

\section{Keywords:}

1. Magnetic Resonance Imaging

2. Support Vector Machine

3. Autistic Disorder

*Corresponding Author: Hadiseh Hasani

Email:h.hasani@tafreshu.ac.ir 


\title{
بكارگيرى كاهش ابعاد در تصاوير fMRI بر اساس الكوريتم فر البتكارى جهت تشخيص اوتيسم
}

\author{
فرزانه صادقيان، حديثه حسنى"، مرضيه جعفرى
}

كروه زئودزى و مهيندسى نقشه بردارى، دانشخاه تفرش، تفرش، ايران

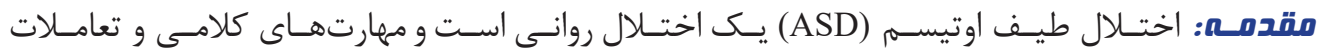

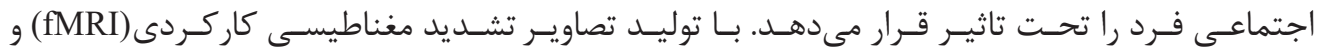

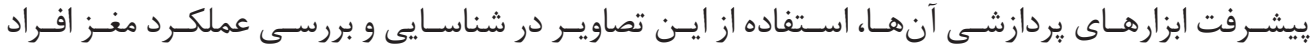

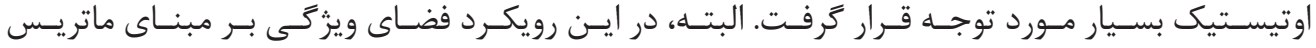

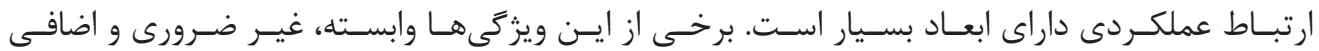

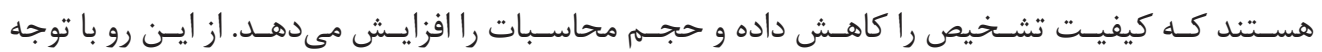

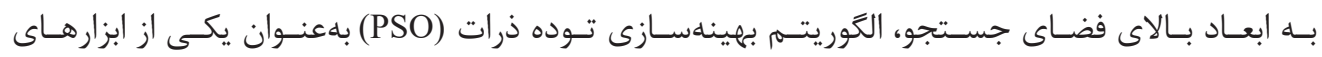

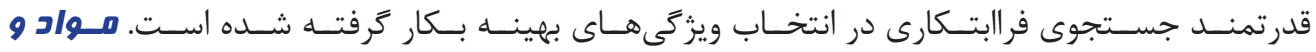

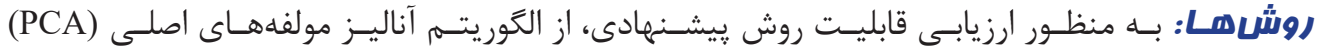

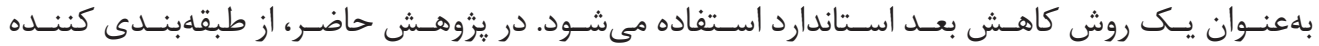

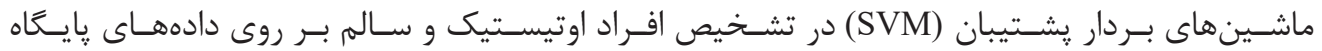

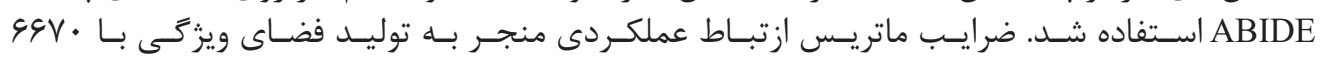

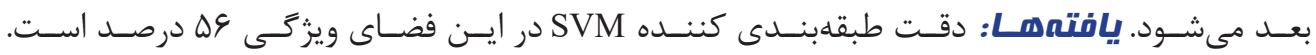

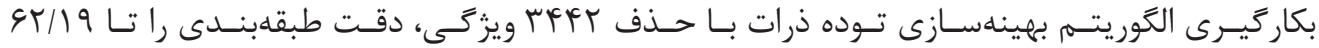

وازههاى كليدى:

1

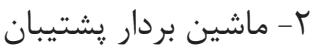
r- اختلال اوتيسن

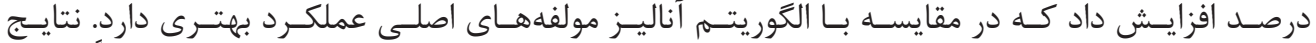

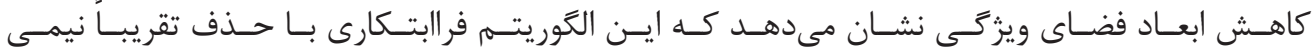

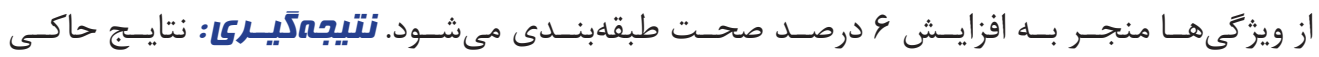

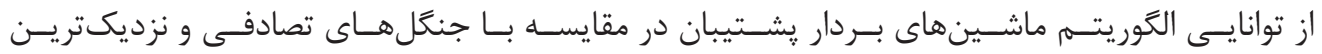

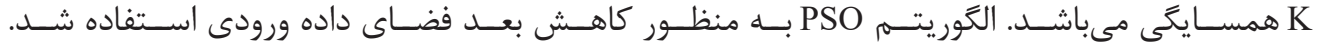




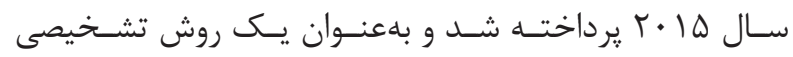

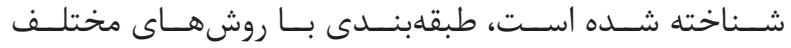

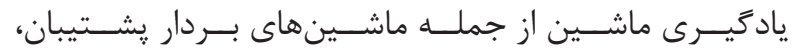

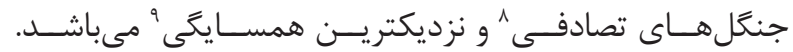

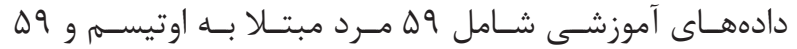

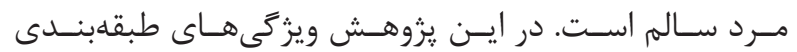

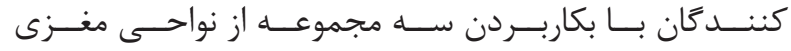

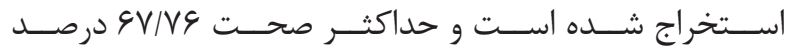

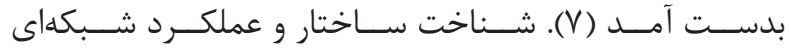

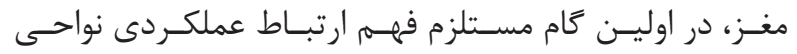

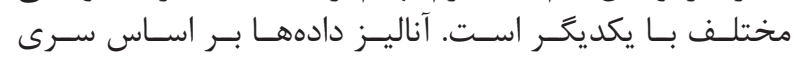

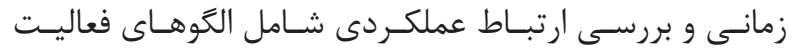

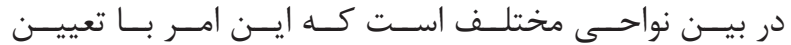

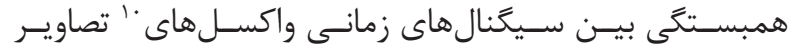

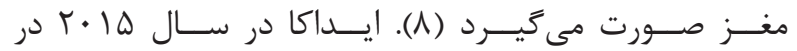

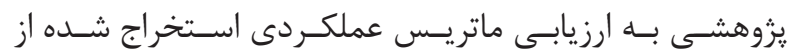

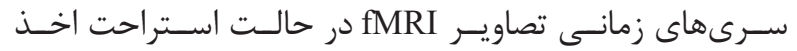

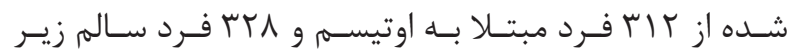

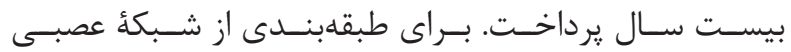

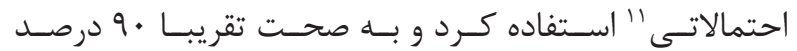

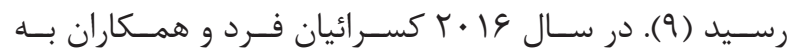

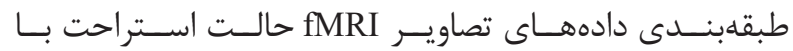

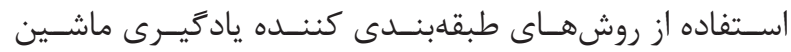

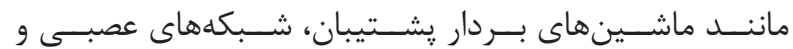

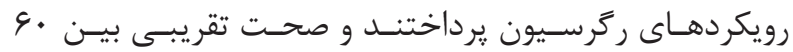

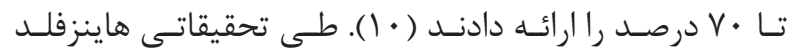

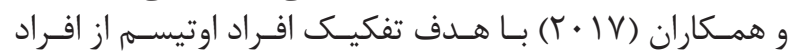

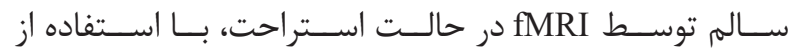

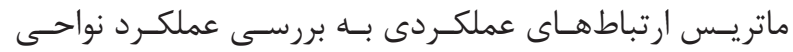

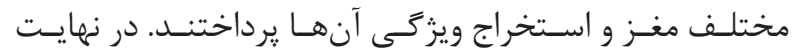

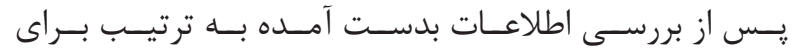

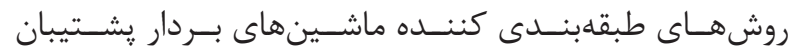

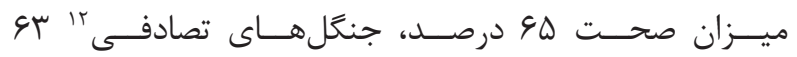

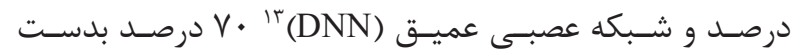

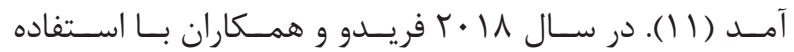

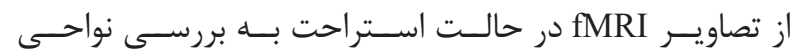

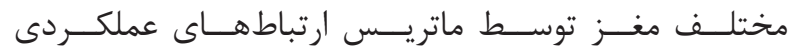

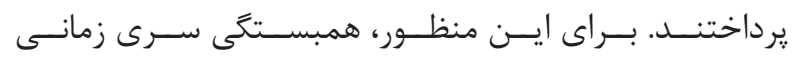

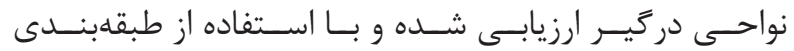

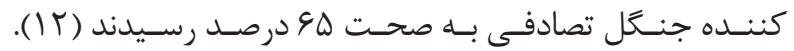

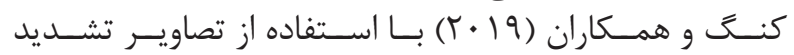

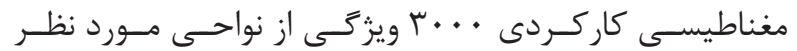

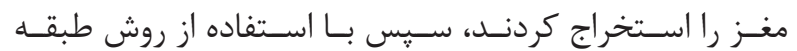

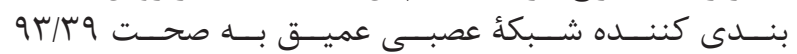

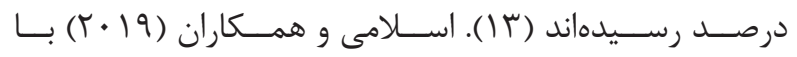

${ }^{1}$ Brain Mapping

${ }^{2}$ Task

${ }^{3}$ Rest

${ }^{4}$ Blood Oxygenation Level Dependent (BOLD)

${ }^{5}$ Prefrontal Cortex

${ }^{6}$ Temporal Cortex

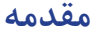

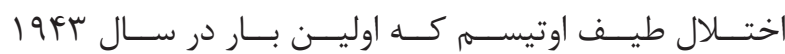

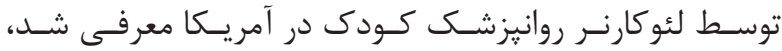

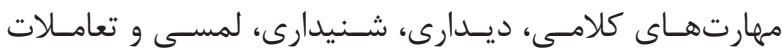

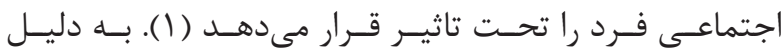

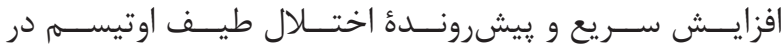

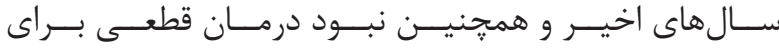

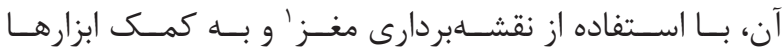

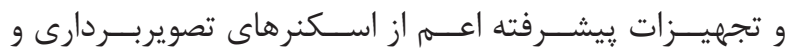

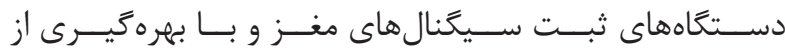

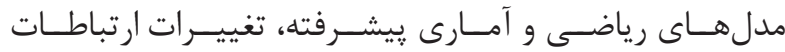

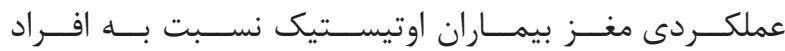

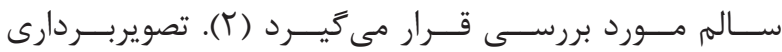

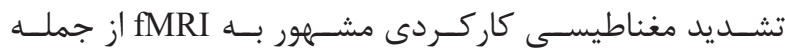

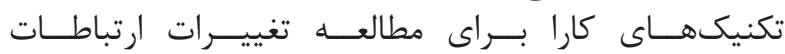

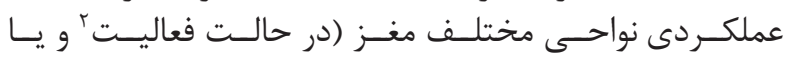

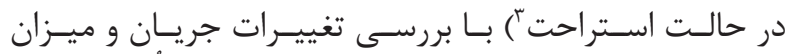

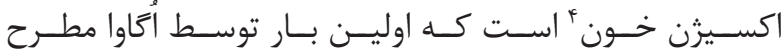

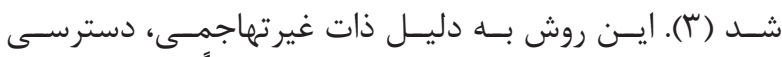

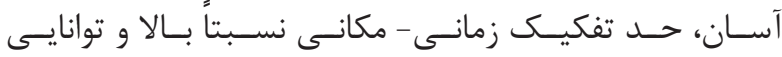

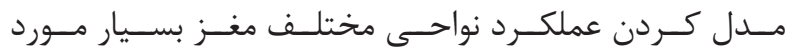

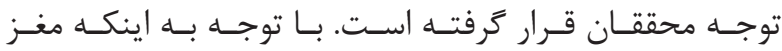

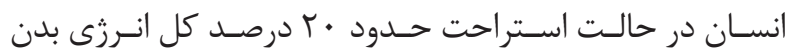

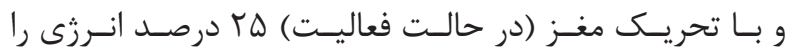

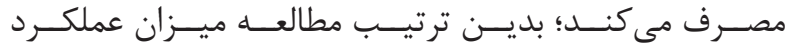

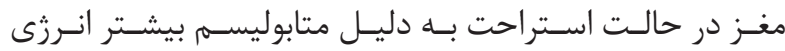

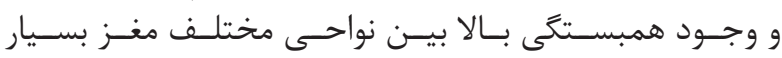

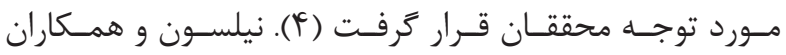

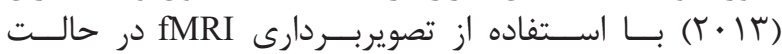

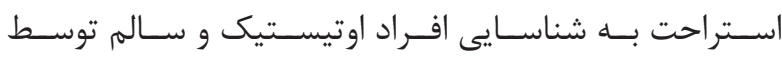

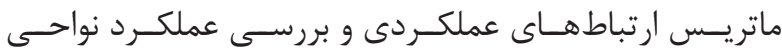

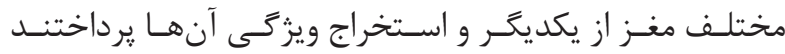

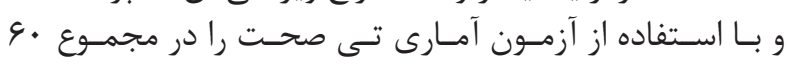

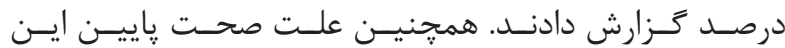

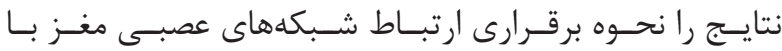

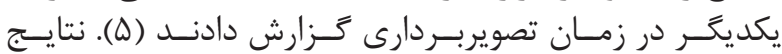

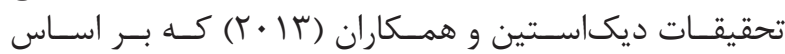

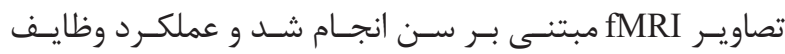

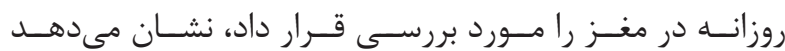

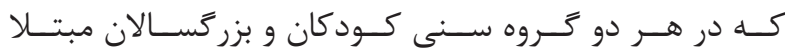

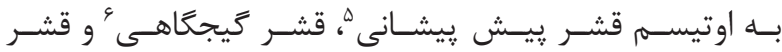

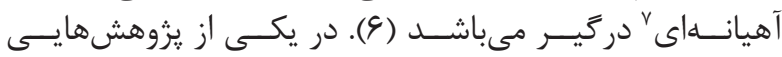

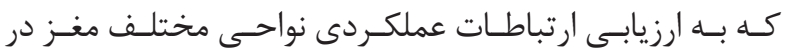

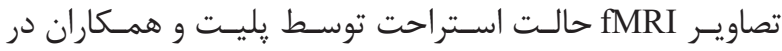

\footnotetext{
${ }^{7}$ Parietal Cortex

${ }^{8}$ Random Forests

${ }^{9} \mathrm{KNN}$ : K-Nearest Neighbor

${ }^{10}$ Voxel

${ }^{11}$ Probabilistic Neural Network (PNN)

${ }^{12}$ Random Forests

${ }^{13}$ Deep Neural Network
} 


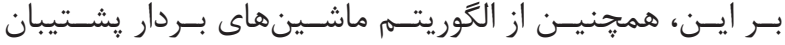

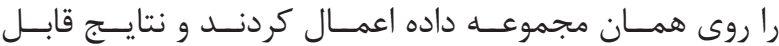

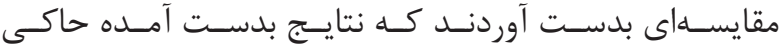

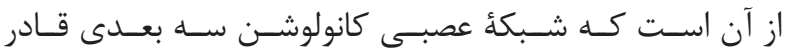

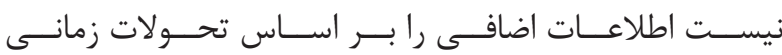

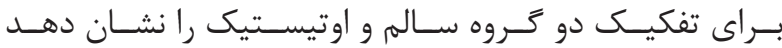

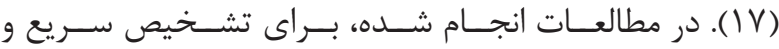

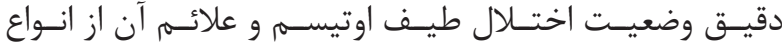

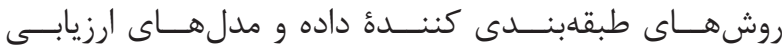

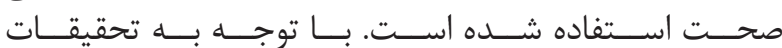

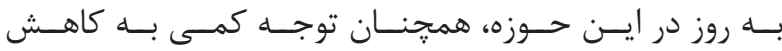

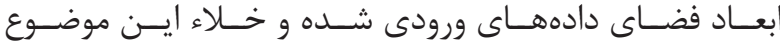

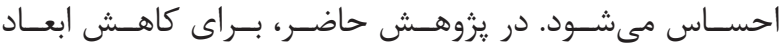

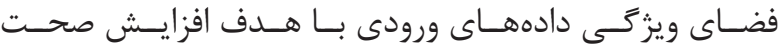

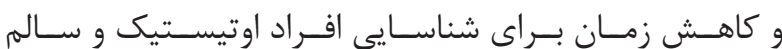

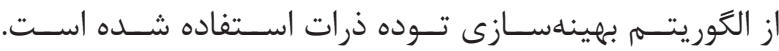

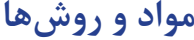

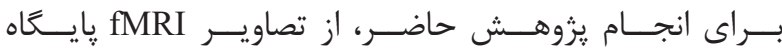

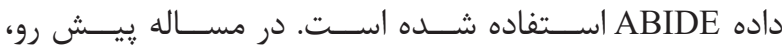

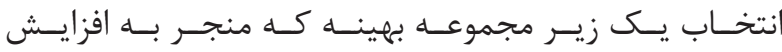

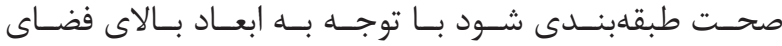

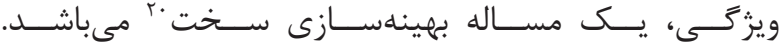

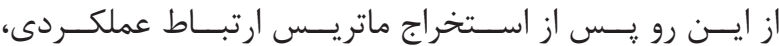

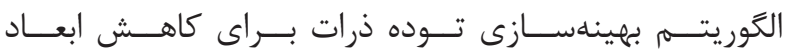

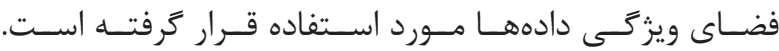

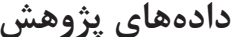

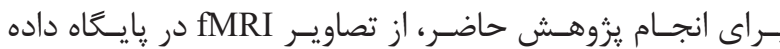

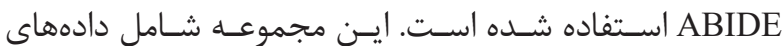

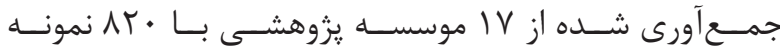

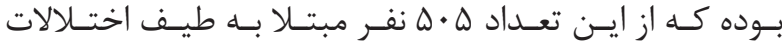

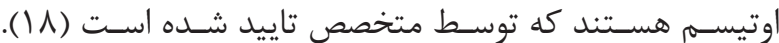

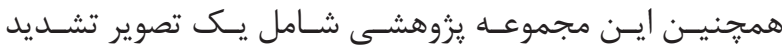

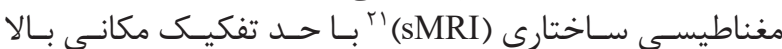

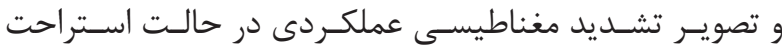

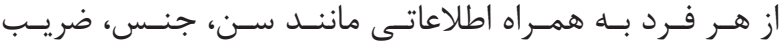

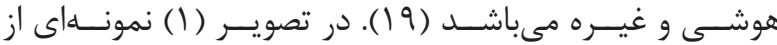

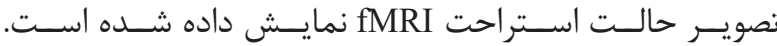

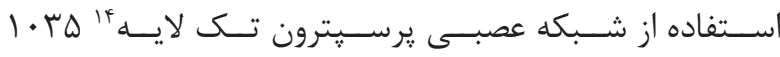

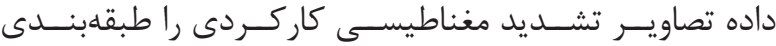

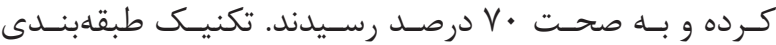

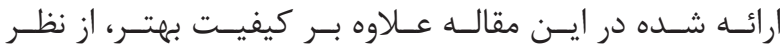

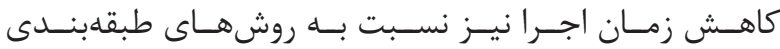

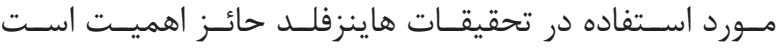

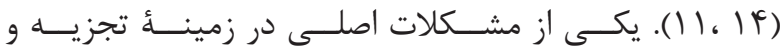

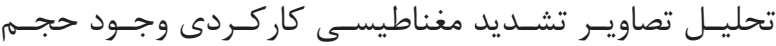

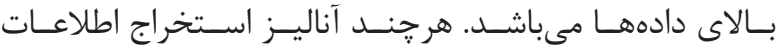

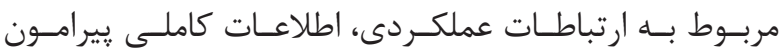

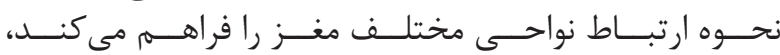

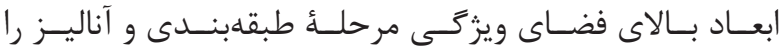

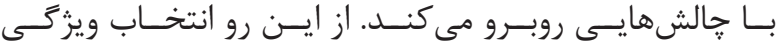

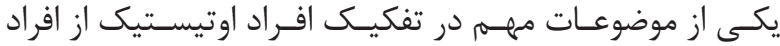

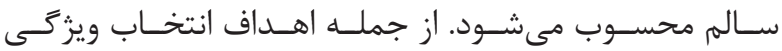

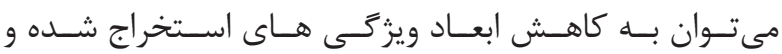

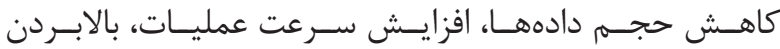

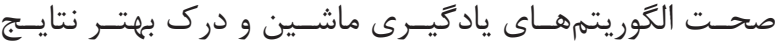

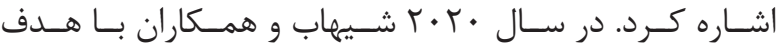

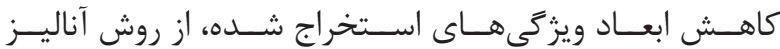

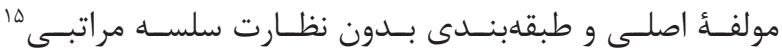

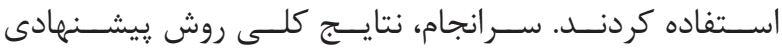

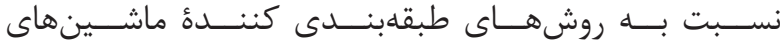

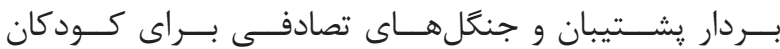

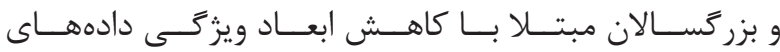

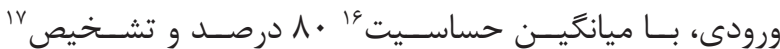

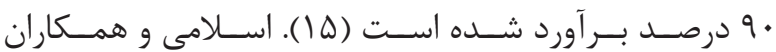

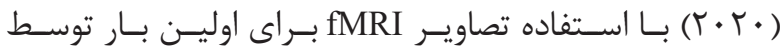

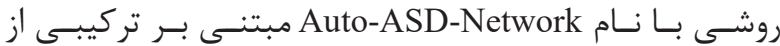

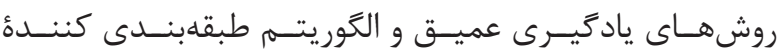

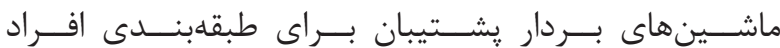

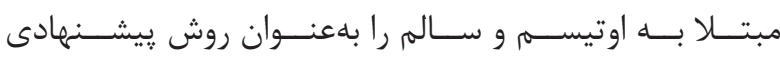

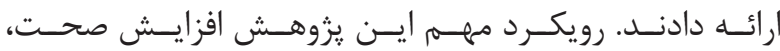

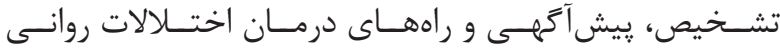

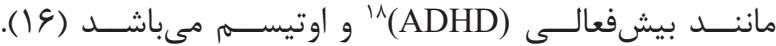

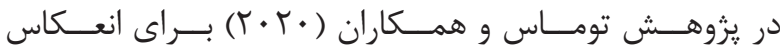

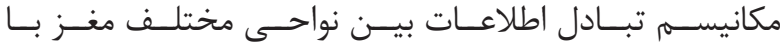

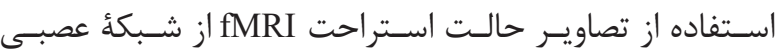

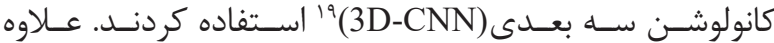

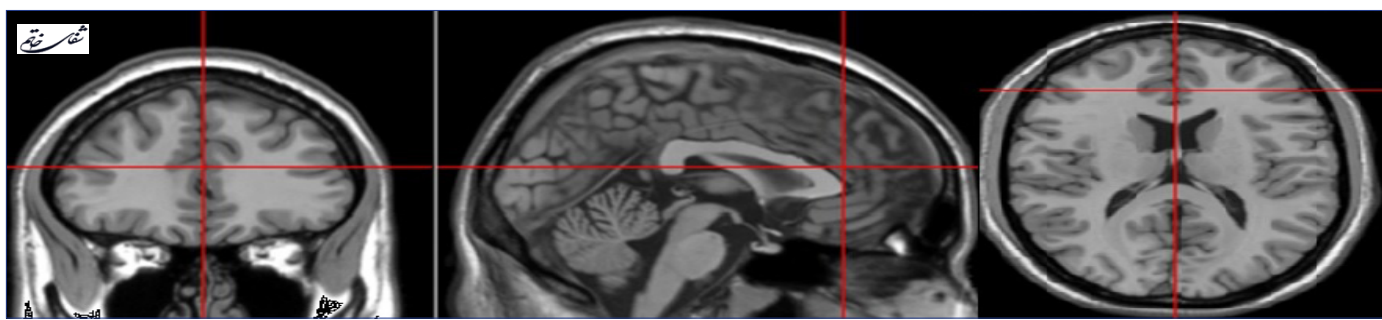

fMRI تصوير 1- تصوير حالت استراحت
${ }^{14}$ ASD-DiagNet

${ }^{15} \mathrm{HC}$ : Hierarchical Classification

16 Sensitivity

${ }^{17}$ Specificity
${ }^{18}$ Attention Deficit Hyperactivity Disorder (ADHD)

19 Three-Dimensional Convolutional Neural Network

20 NP-hard

${ }^{21}$ Structural Magnetic Resonance Imaging (sMRI) 


\section{fMRI بيشيردازش تصاوير}

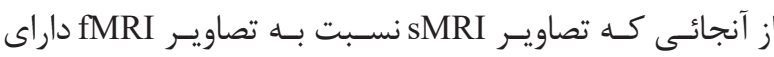

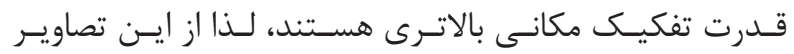

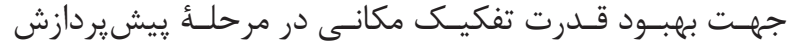

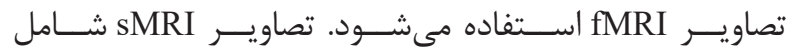

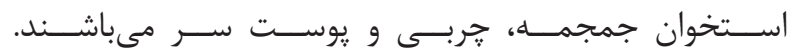

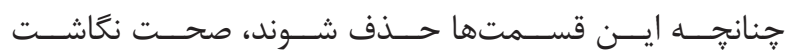

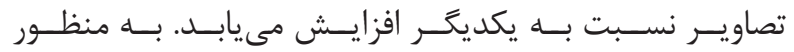

روش پِيشنهادى

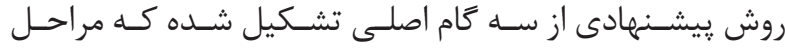

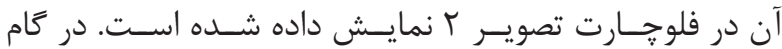

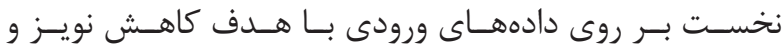

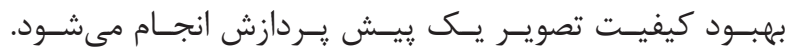

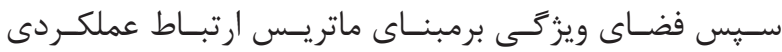

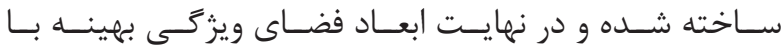

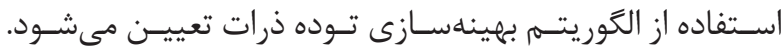

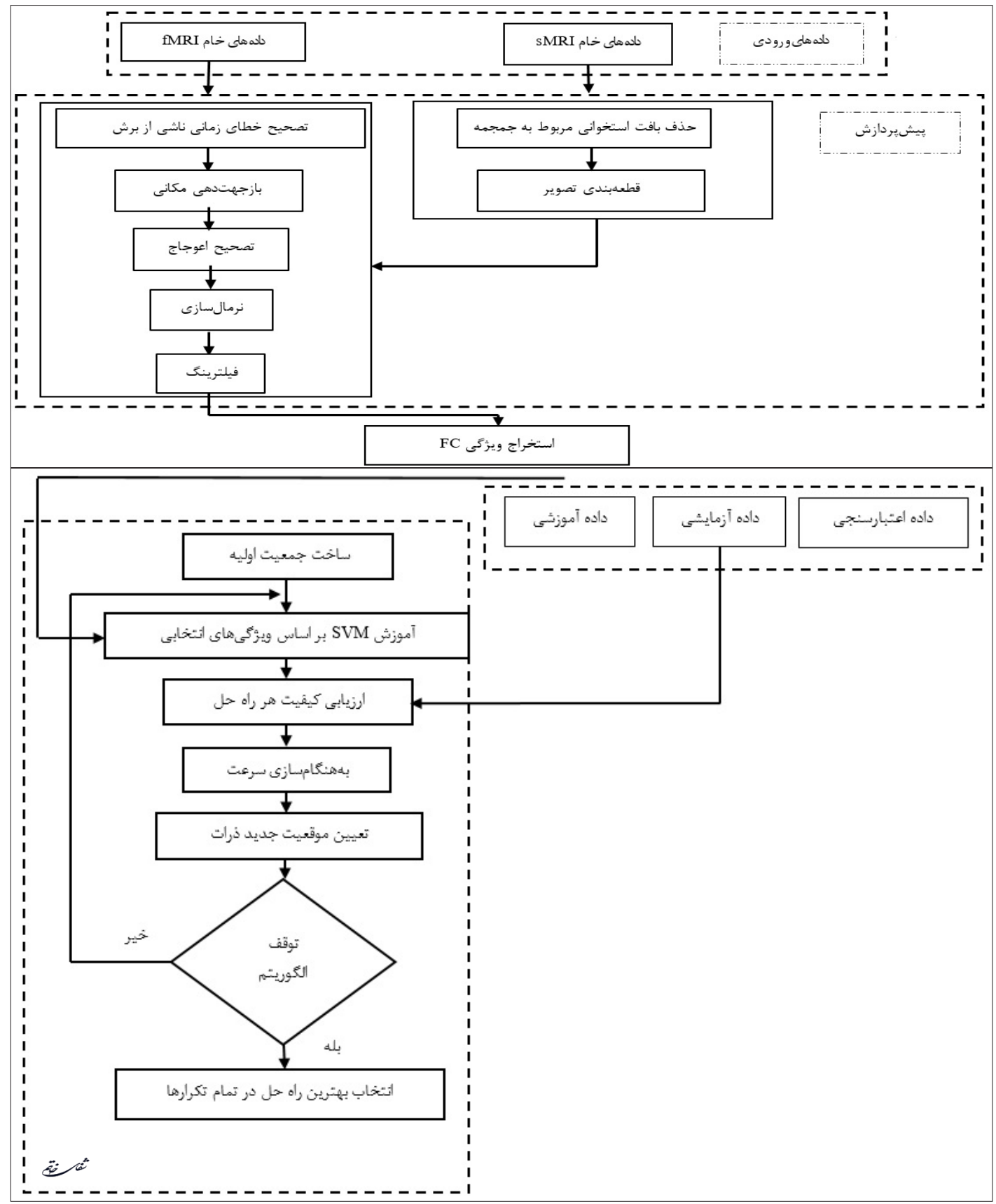




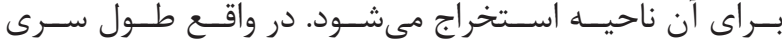

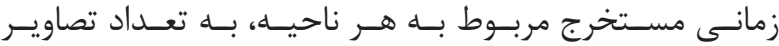

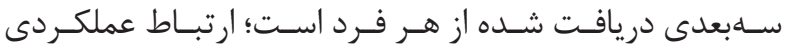

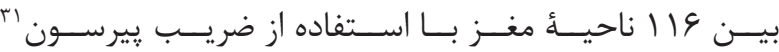

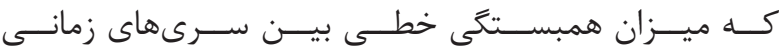

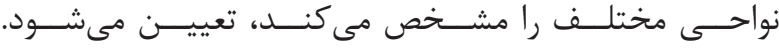

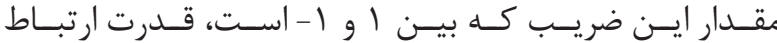

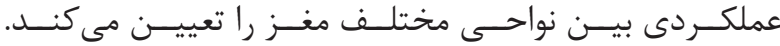

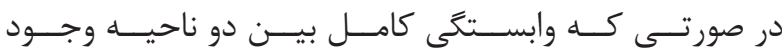

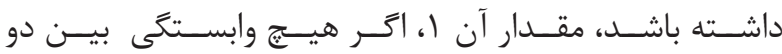

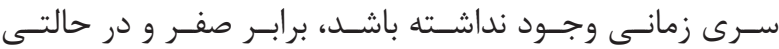

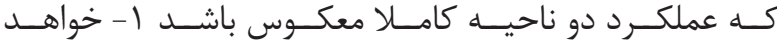

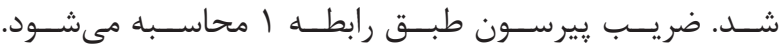

(1) $r_{i j}=\frac{\sum_{t=1}^{n}\left[\left[x_{i}(t)-\bar{x}_{i}\right]\left[x_{j}(t)-\bar{x}_{j}\right]\right)}{\sqrt{\sum_{t=1}^{n}\left[x_{i}(t)-\bar{x}_{i}\right]^{2}} \sqrt{\sum_{t=1}^{n}\left[x_{j}(t)-\bar{x}_{j}\right]^{2}}}$

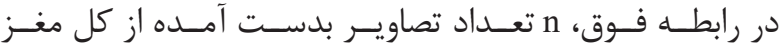

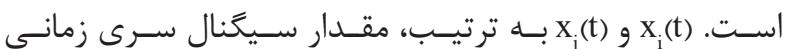

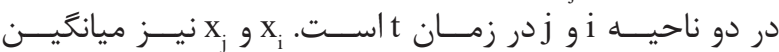

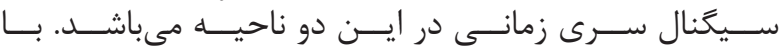

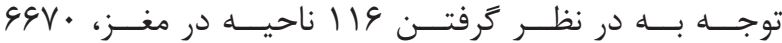

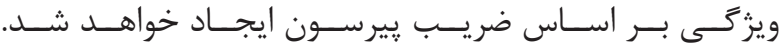

كاهش ابعاد مبتنى بر الكوريتهم بهينهسازى توده ذرات

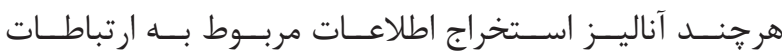

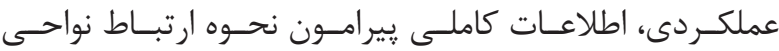

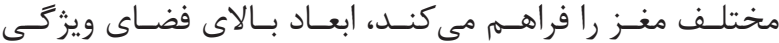

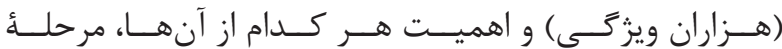

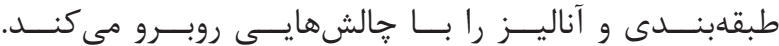

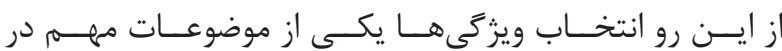

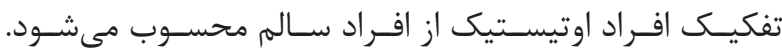

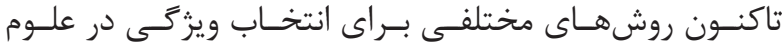

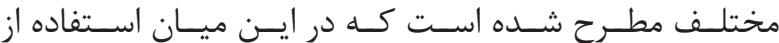

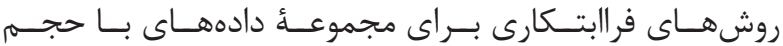

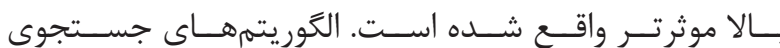

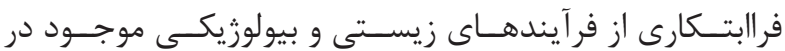

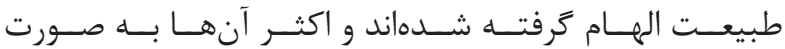

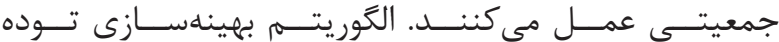

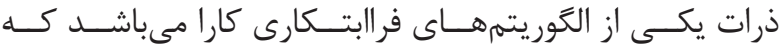

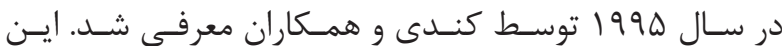

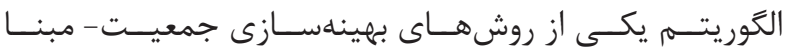

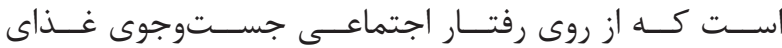

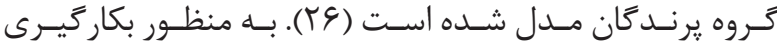

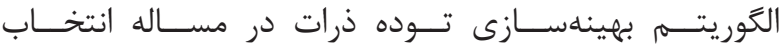

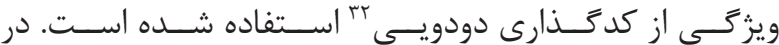

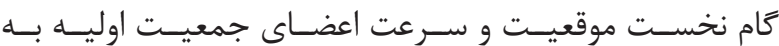

\footnotetext{
${ }^{22}$ Slice Timing Correction

${ }^{23}$ Spatial Realignment

${ }^{24}$ Distortion Correction

${ }^{25}$ Normalization

${ }^{26}$ Affine

${ }^{27}$ Spatial Smoothing
}

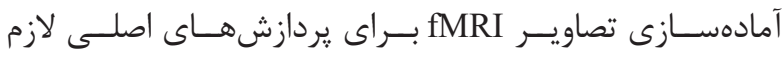

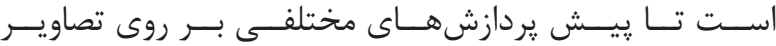

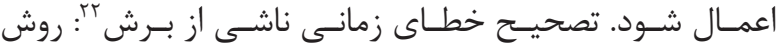

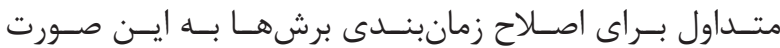

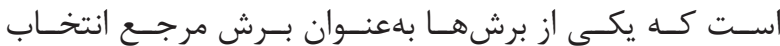

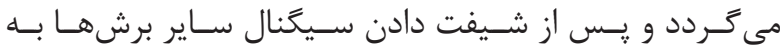

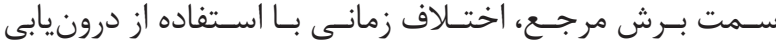

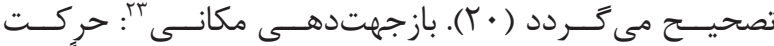

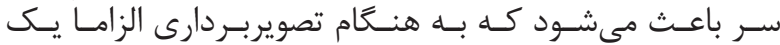

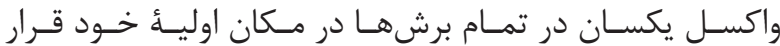

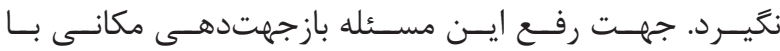

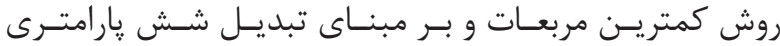

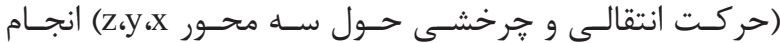

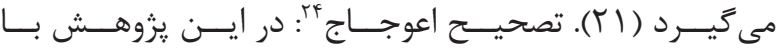

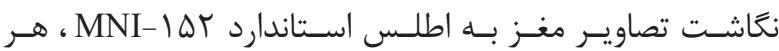

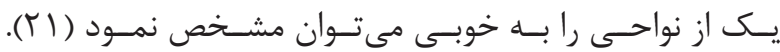

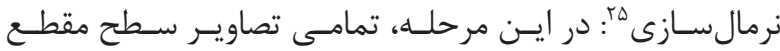

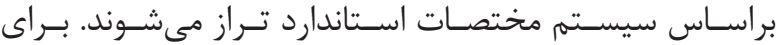

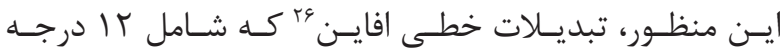

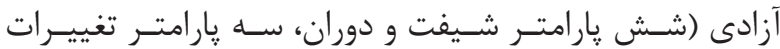

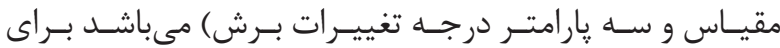

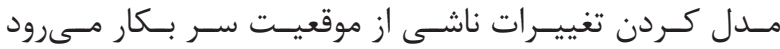

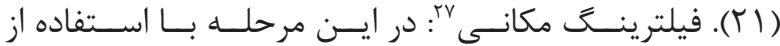

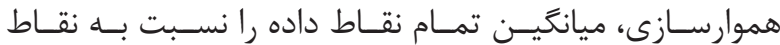

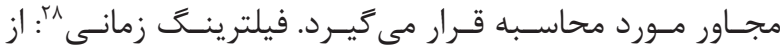

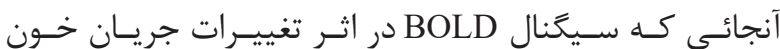

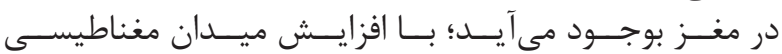

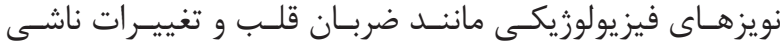

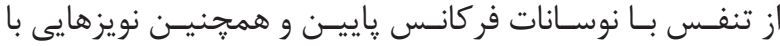

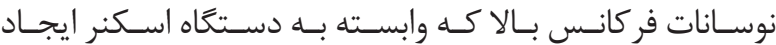

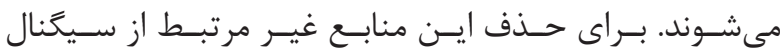

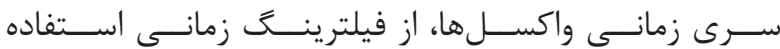

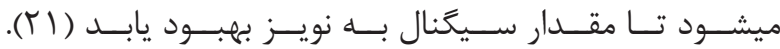

\section{استخراج ويزگى بر اساس ارتباط عملكردى}

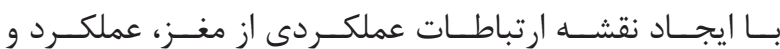

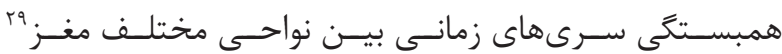

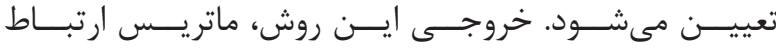

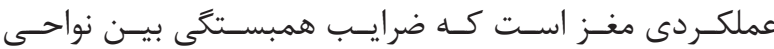

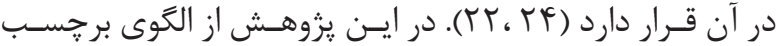

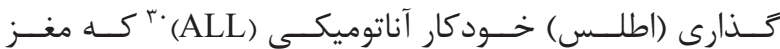

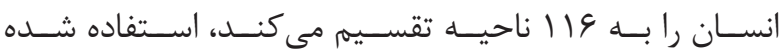

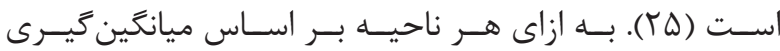

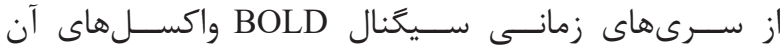

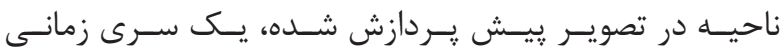

\footnotetext{
${ }^{28}$ Temporal Filtering

${ }^{29}$ Region of Interest(ROI)

${ }^{30}$ Automated Anatomical Labeling

${ }^{31}$ Pearson Correlation Coefficient

${ }^{32}$ Binary Coding
} 


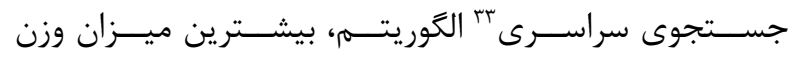

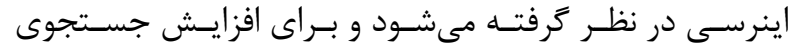

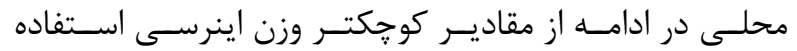

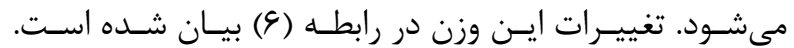
(द) $w=w_{\max }-t \times\left(\left(w_{\max }-w_{\min }\right) / M a x_{i t e r}\right)$

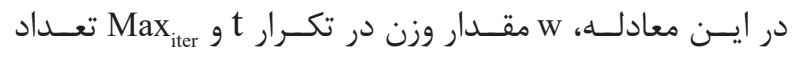

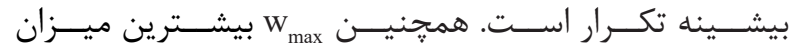

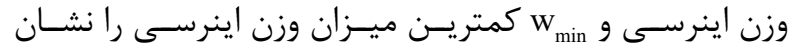

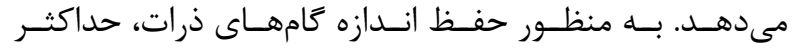

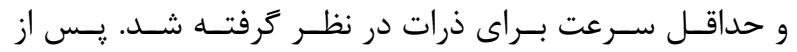

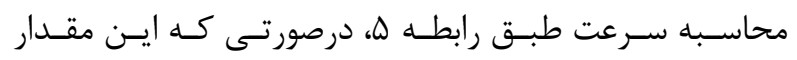

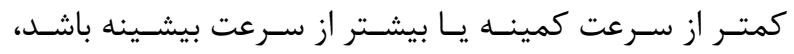

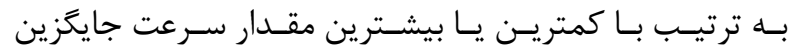

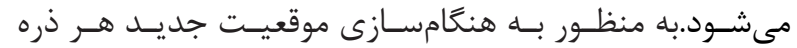

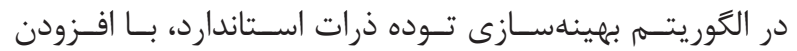

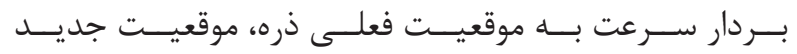

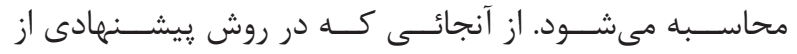

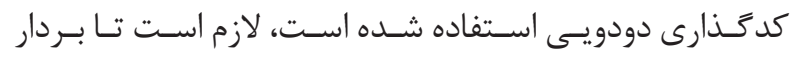

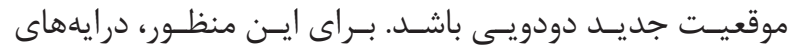

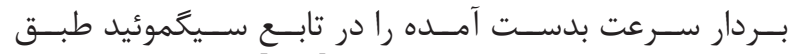

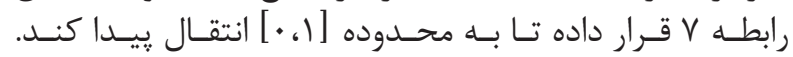

$$
\mathrm{S}\left(\mathrm{V}_{\mathrm{ij}}\right)=\frac{1}{1+\exp \left(-\mathrm{V}_{\mathrm{ij}}\right)}
$$

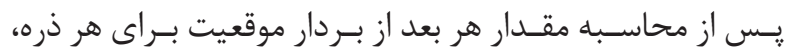

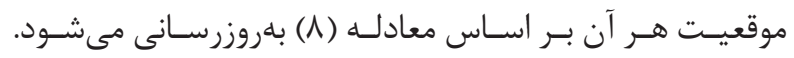

$$
\text { (ᄉ) } \quad X_{i j}=\left\{\begin{array}{lr}
1 & p_{i j}<S\left(V_{i j}\right) \\
0 & \text { otherwise }
\end{array}\right.
$$

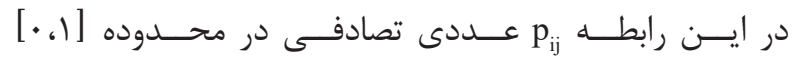

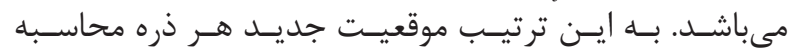

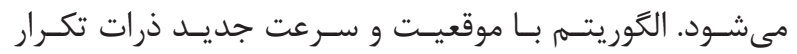

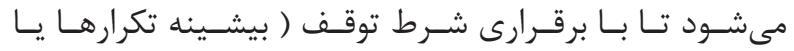

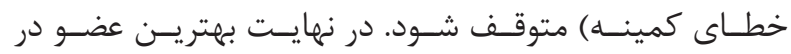

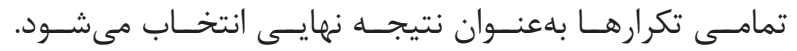

\section{يافتهها}

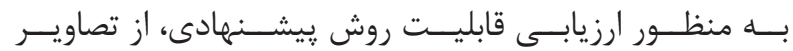
يايـحًاه داده ABIDE fMRI

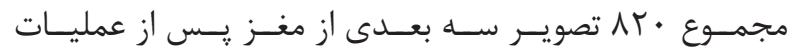

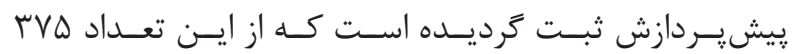

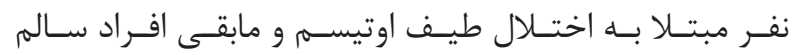

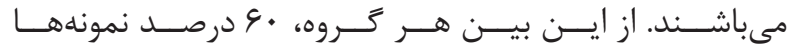

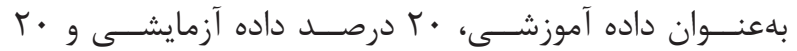

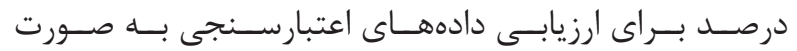

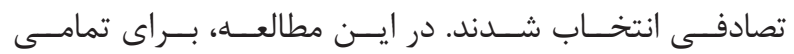

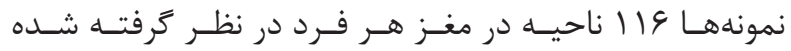

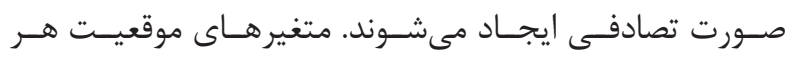

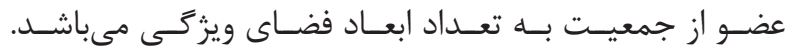

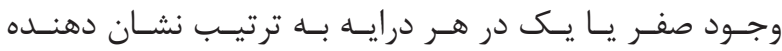

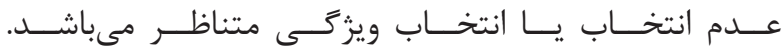

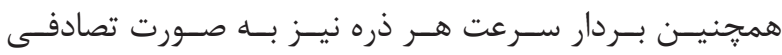

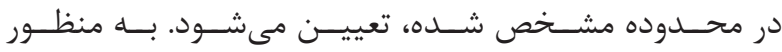

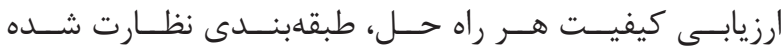

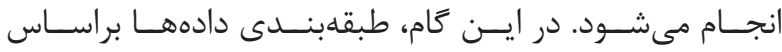

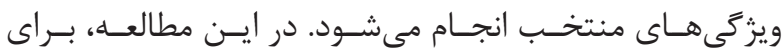

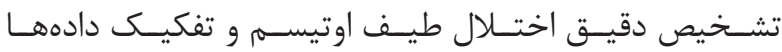

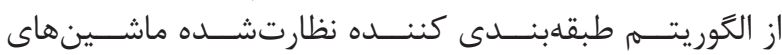

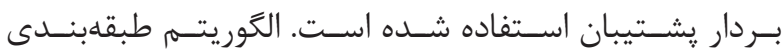

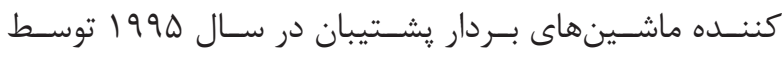

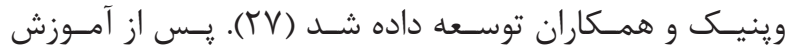

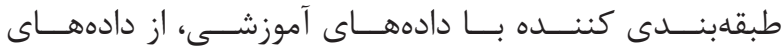

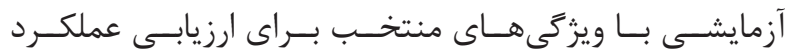

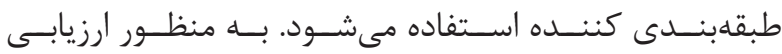

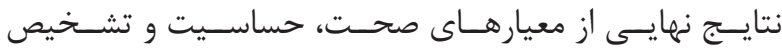

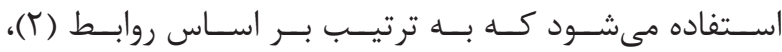

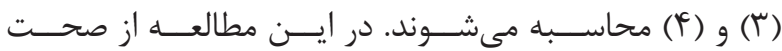

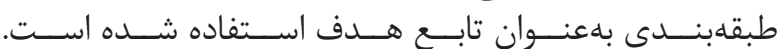
Accuracy $=(\mathrm{TP}+\mathrm{TN}) /(\mathrm{TP}+\mathrm{TN}+\mathrm{FP}+\mathrm{FN})$ Sensitivity $=\mathrm{TP} /(\mathrm{TP}+\mathrm{FN})$ Specificity $=\mathrm{TN} /(\mathrm{FP}+\mathrm{TN})$

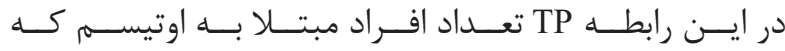

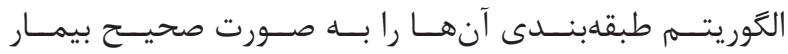

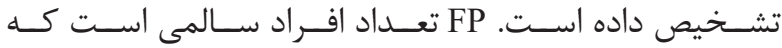

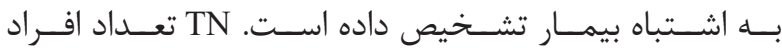

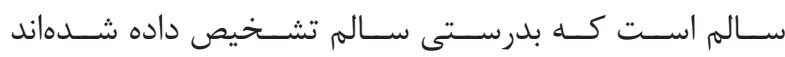

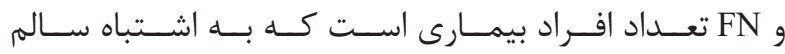

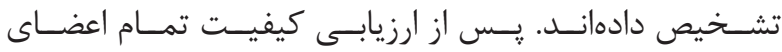

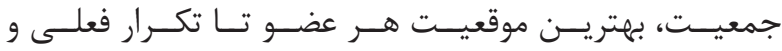

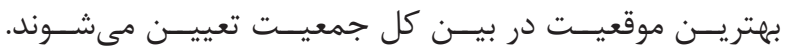

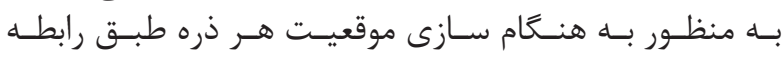

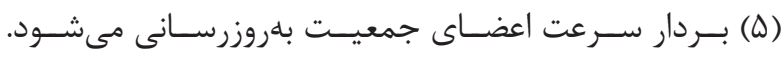
(ఏ) $V_{\mathrm{ij}}(\mathrm{t})=\mathrm{w} \times \mathrm{V}_{\mathrm{ij}}(\mathrm{t}-1)+\mathrm{c}_{1} \times \mathrm{r}_{1} \times\left(\mathrm{p}_{\text {best }}-\mathrm{X}_{\mathrm{ij}}(\mathrm{t}-1)+\mathrm{c}_{2} \times \mathrm{r}_{2} \times\left(\mathrm{g}_{\text {best }}-\mathrm{X}_{\mathrm{ij}}(\mathrm{t}-1)\right)\right.$

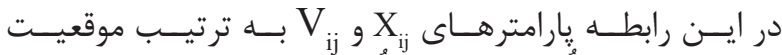

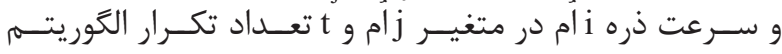

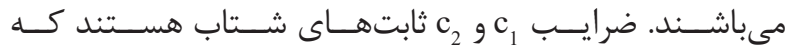

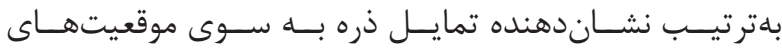

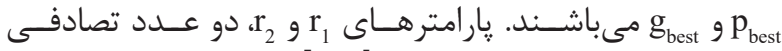

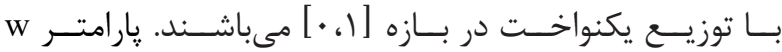

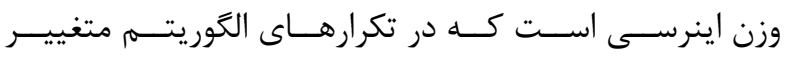

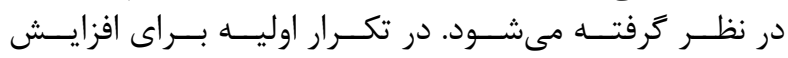


بــر روى دادههــاى ورودى در جــدول آ آورده شــده اسـت.

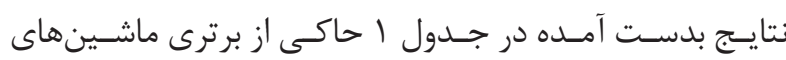

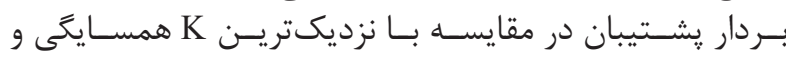

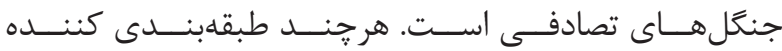

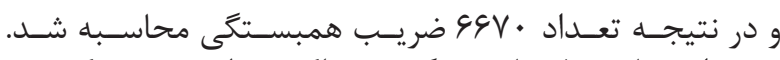

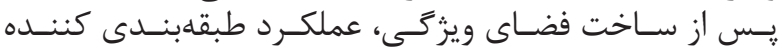

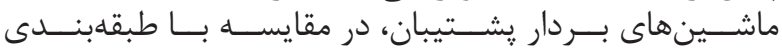

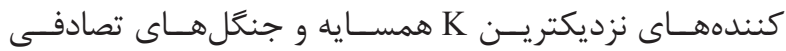

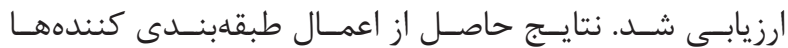

جدول ا- مقايسٔ طبقدبندى كنندهها

\begin{tabular}{|c|c|c|c|c|c|c|}
\hline \multicolumn{3}{|c|}{ داده اعتبارسنجى } & \multicolumn{3}{|c|}{ داده تست } & \multirow{2}{*}{ نوع طبقهبندى كننده } \\
\hline تشخيص & حساسيت & صحت & تشخيص & حساسيت & صحت (درصد) & \\
\hline .199 & $\cdot 109$ & $9 \cdot 19 \mathrm{~V}$ & - $/ 0 T$ & $\cdot 109$ & $\Delta q / \cdot 9$ & ماشينهاى بردار رشتيبان \\
\hline$\cdot / 4$ & $.19 \mathrm{~V}$ & $\Delta Q / \uparrow \Lambda$ &.$/ 49$ & $\cdot / V T$ & DI/AT & نزديكترين همسايگى \\
\hline .109 & . /QH & $\Delta F / A V$ & $.10 \mid$ & .194 & $\Delta V / T I$ & جنكل هاى تصادفى \\
\hline
\end{tabular}

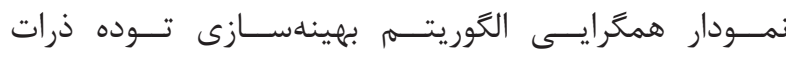

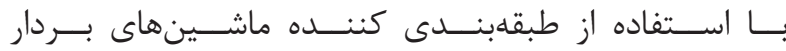

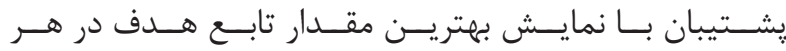

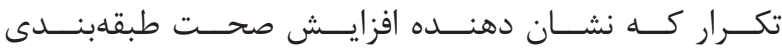

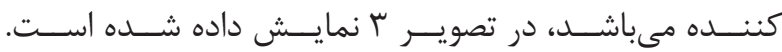

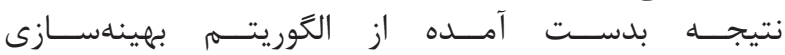

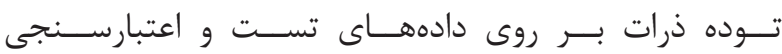

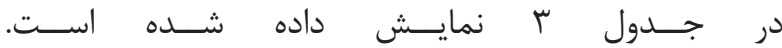

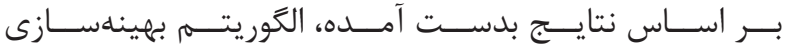

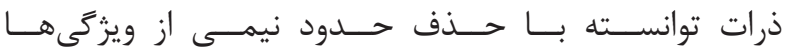

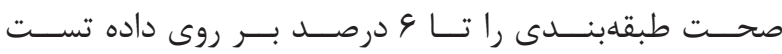

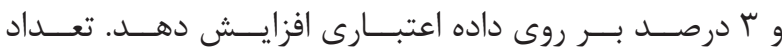

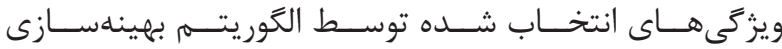

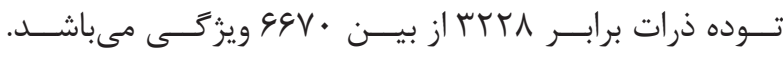

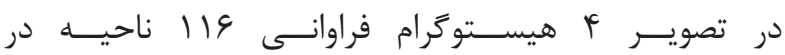

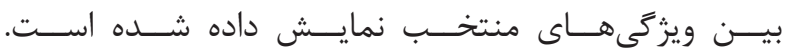

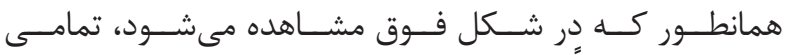

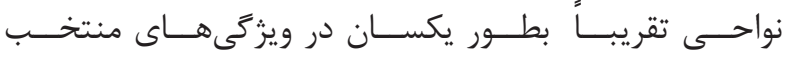

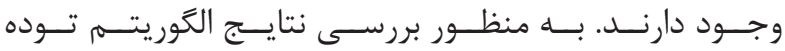

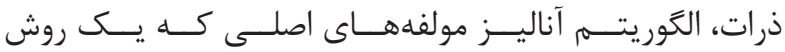

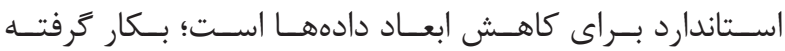

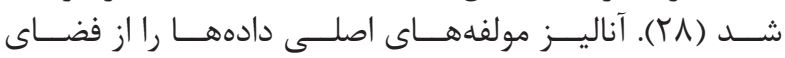

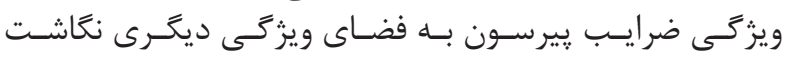

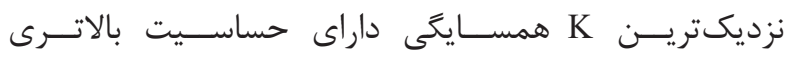

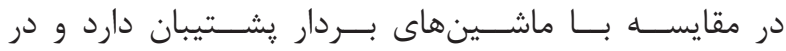

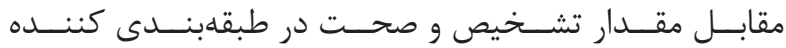

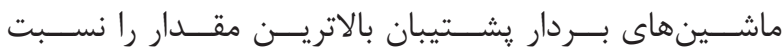

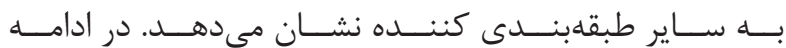

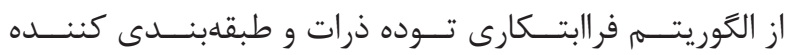

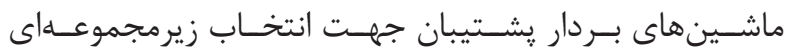

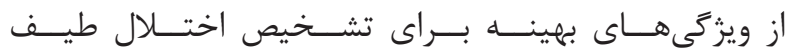

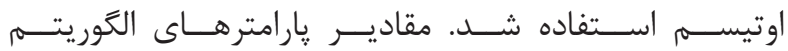

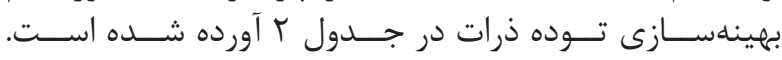

جدول r- مقادير بارامترهاى الكوريتم بهينهسازى توده ذرات

\begin{tabular}{|c|c|}
\hline مقدار & 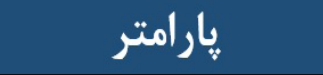 \\
\hline $99 \mathrm{~V}$. & ابعاد هر ذره \\
\hline 1 . & تعداد اعضاى جمعيت \\
\hline $1 \cdots$ & حداكثر تكرار \\
\hline$(-Y, r)$ & محدوده مجاز سرعت \\
\hline$(\cdot / \cdot, p / q)$ & محدوده تغييرات W \\
\hline r & $\mathrm{c}_{1}$ \\
\hline r & $\mathrm{c}_{2}$ \\
\hline
\end{tabular}

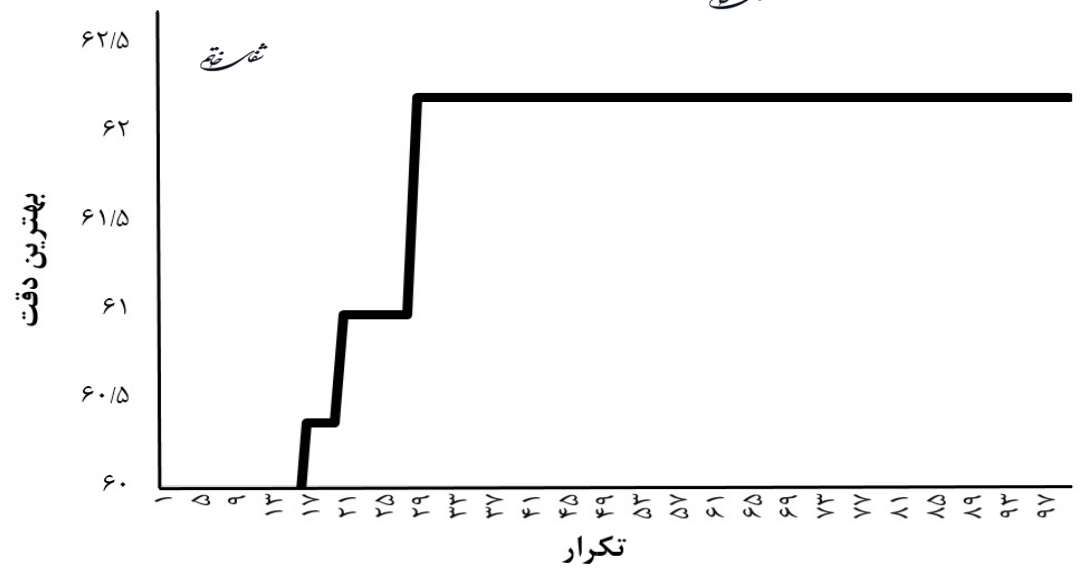


جدول r - مقايسٔ طبقهبندى كنندهها

\begin{tabular}{|c|c|c|c|c|c|c|c|}
\hline \multicolumn{3}{|c|}{ داده اعتبارسنجى } & \multicolumn{3}{|c|}{ داده تست } & \multirow{2}{*}{ تعداد ويثركىها } & \multirow{2}{*}{ نوع طبقهبندى كننده } \\
\hline تشخيص & حساسيت & صحت & تشخيص & حساسيت & صحت (درصد) & & \\
\hline .199 & . 109 & $9.19 \mathrm{~V}$ & $\cdot \mid a r$ & $\cdot / 109$ & $\Delta 9 / .9$ & $99 \%$. & ماشينهاى بردار يُشتيبان \\
\hline$\cdot|N|$ & .194 & $99^{\mu / 4}$ & $\cdot \mid \Delta \Lambda$ & .190 & $94 / 19$ & rYYA & نزيكترين همسايكى \\
\hline
\end{tabular}

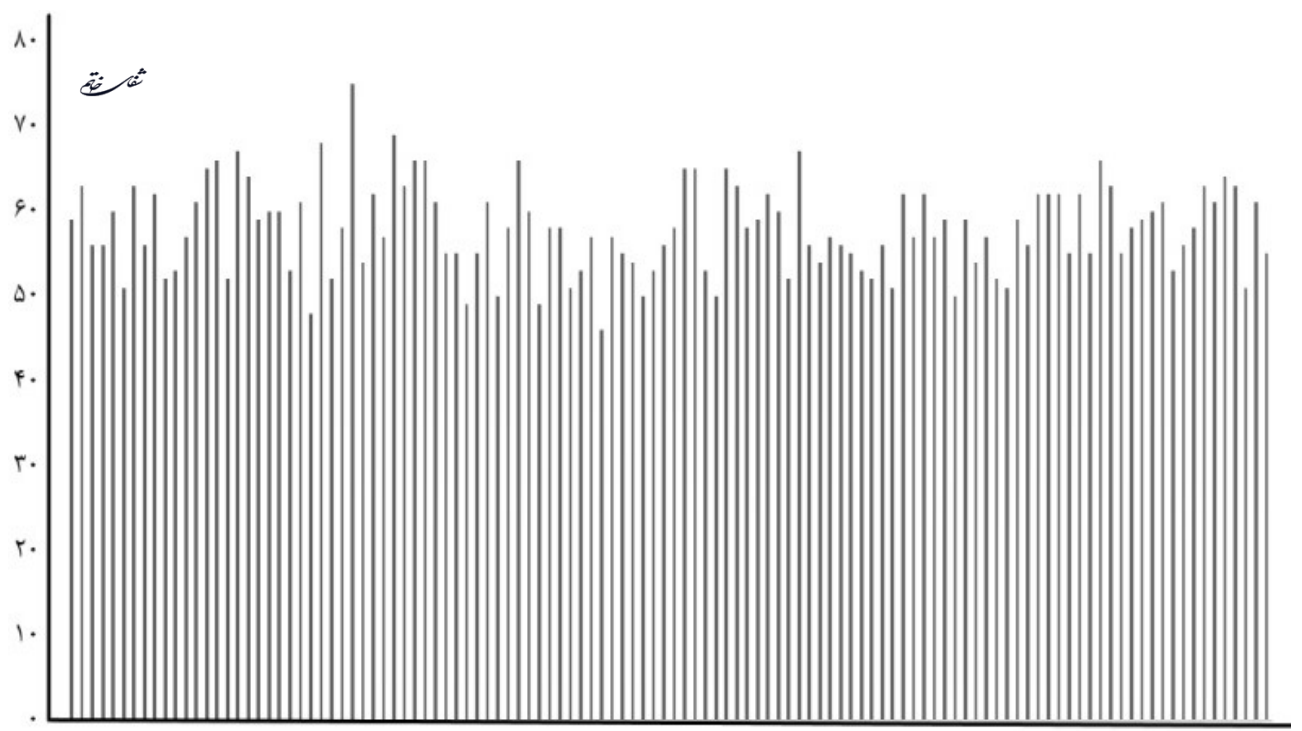

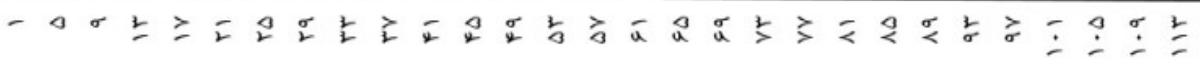

تصوير F- هيستوكرام فراوانى 19 إ ناحيه در ويثَى هاى منتخب

آمـــده از الكوريتــهم بهينهســازى تــوده ذرات مقايســهـ شــد.

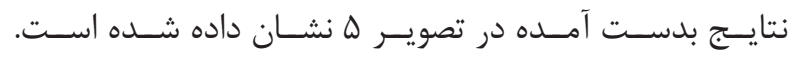

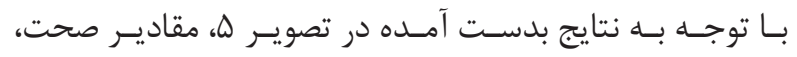

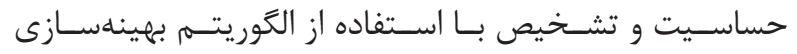

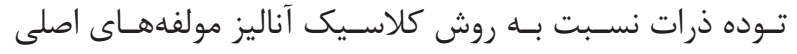

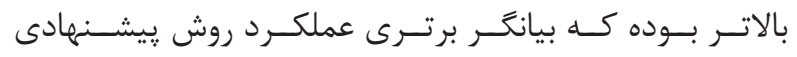

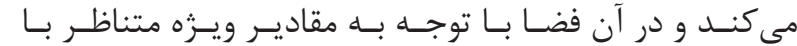

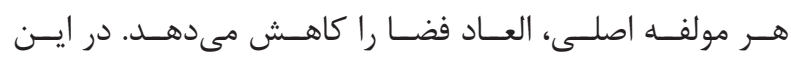

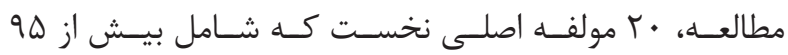

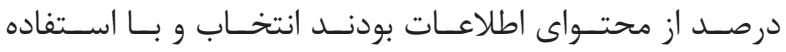

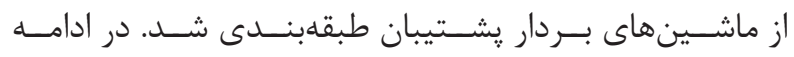

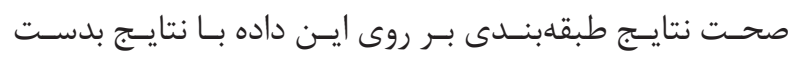

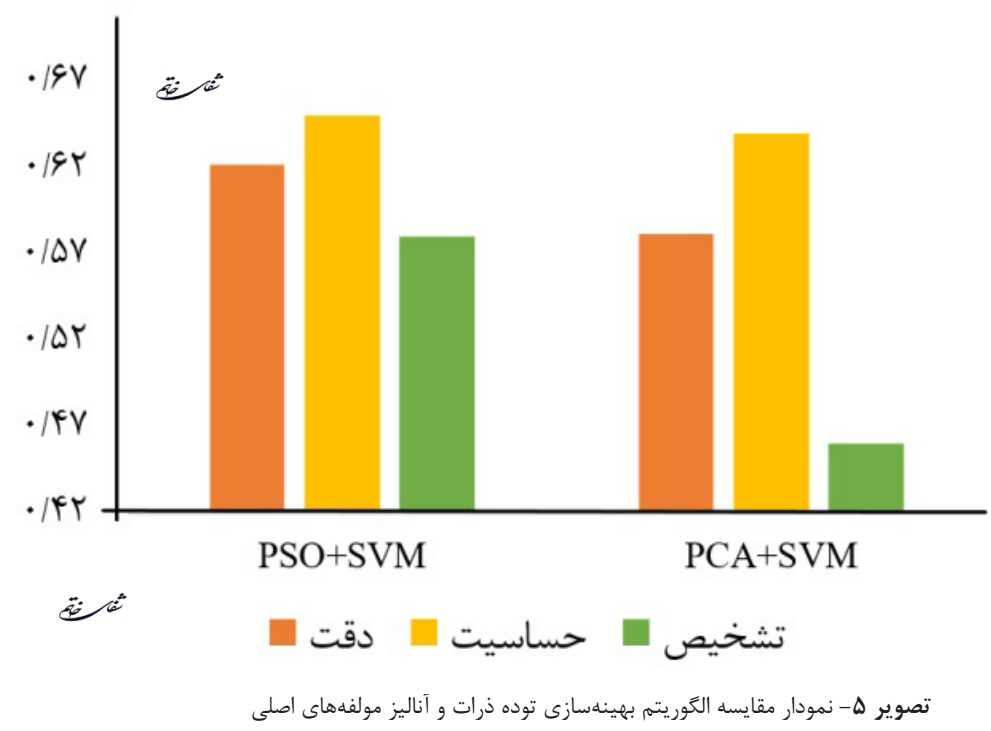




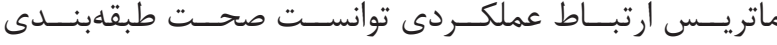

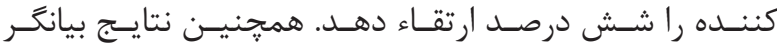

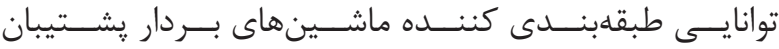

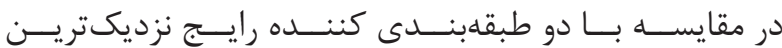

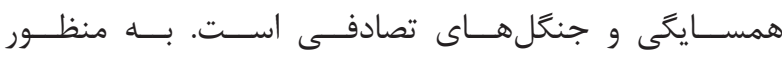

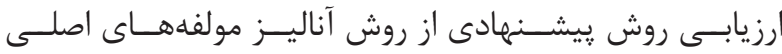

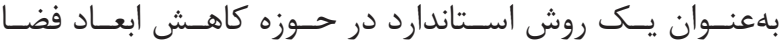

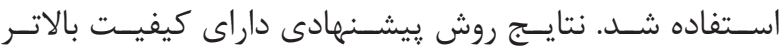

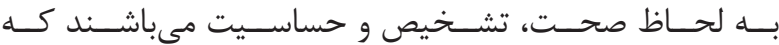

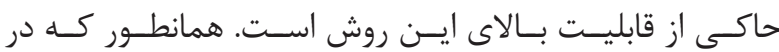

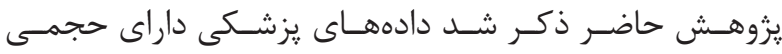

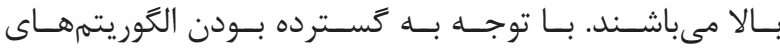

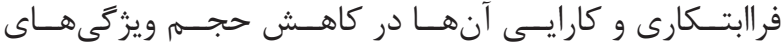

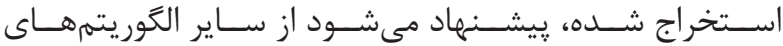

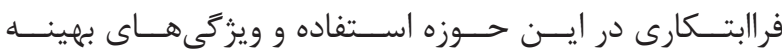

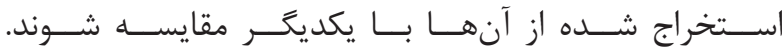

1. Ghaffari M.A, Mousavinejad. E, Riahi. F, Mousavinejad. M, Afsharmanesh M.R. Increased Serum Levels of Tumor Necrosis Factor-Alpha, Resistin, And Visfatin in the Children with Autism Spectrum Disorders: A Case-Control Study. Hindawi Publishing Corporation; Article ID 9060751 2016.; pp: 7.

2. Savoy R.L. History and Future Directions of Human Brain Mapping and Functional Neuroimaging. Elsevier Science B.V 2001; pp: 9-42.

3. Ogawa. S. Magnetic Resonance Imaging of Blood Vessels at High Fields: in Vivo and in Vitro Measurements and Image Simulation. Magnetic Resonance Imaging 1990; vol.16, No.1. pp: 9-18.

4. Fox M.D and Raichle M.E. Spontaneous Fluctuations in Brain Activity Observed with Functional Magnetic Resonance Imaging. Nature 2007; vol. 8, pp: 700-711.

5.NielsenJ.A,ZielinskiB.A,FletcherP.T,AlexanderA.L, Lange.N,BiglerE.D, et.MultisiteFunctionalConnectivity MRI Classification of Autism: ABIDE Results. Brain a Journal of Neurology 2013; pp: 134: 3742-3754.

6. Dickstein D.P, Pescosolido M.F, Reidy B.L, Galvan. T, KimK.L, SeymourK.E, et.DevelopmentalMeta-Analysis of the Functional Neural Correlates of Autism Spectrum Disorders. Journal of the American Academy of Child and Adolescent Psychiatry 2013; vol.52, pp: 279-289.

7. Plitt. M, Barnes K.A, Martin. A. Functional

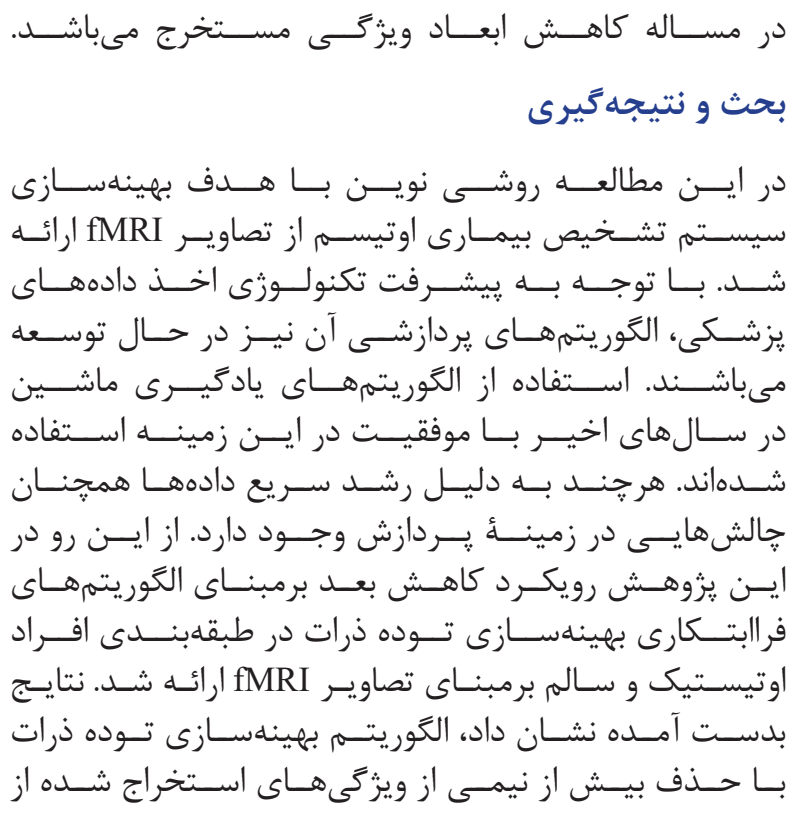

منابع

Connectivity Classification of Autism Identifies Highly Predictive Brain Features but Falls Short of Biomarker Standards. NeuroImage: Clinical 2015; vol.7,pp:359-366.

8. Friston K.J. Functional and Effective Connectivity in Neuroimaging: A Synthesis. Hum. Brain Mapp 1994; vol.2, pp: 56-78.

9. Iidaka. T. Resting State Functional Magnetic Resonance Imaging and Neural Network Classified Autism and Control. Cortex 2015; vol.63, pp: 55-67.

10. Kassraian Fard. P, Matthis. C, Balsters J.H, Maathuis M.H, Wenderoth. N. Promises, Pitfalls, and Basic Guidelines for Applying Machine Learning Classifiers to Psychiatric Imaging Data, with Autism as an Example. Front. Psychiatry 2016; doi: 10.3389/fpsyt.2016.00177.

11. Heinsfeld A.S, Franco A.R, Craddock R.C, Buchweitz. A, Meneguzzi. F. Identification of Autism Spectrum Disorder Using Deep Learning and the ABIDE Dataset. NeuroImage: Clinical 17 2017; pp: 16-23.

12. Fredo A.R.J, Jahedi. A, Reiter. M, Müller R.A. Diagnostic Classification of Autism Using Resting-State fMRI Data and Conditional Random Forest. IEEE; 978-1-5386-3646-6/18/\$31.00, 2018.

13. Kong. Y, Gao. J, Xu. Y, Pan. Y, Wang. J, Liu. J. Classification of Autism Spectrum Disorder by Combining Brain Connectivity and Deep Neural Network Classifier. Neurocomputing 2018; pp: 63-68. 
14. Eslami. T, Mirjalili. V, Fong. A, Laird. A, Saeed. F. ASD-DiagNet: A Hybrid Learning Aapproach for Detection of Autism Spectrum Disorder Using fMRI Data. arXiv:1904.07577v1, 2019.

15. Shihab A.I, Dawood F.A, Kashmar. AH. Data Analysis and Classification of Autism Spectrum Disorder Using Principal Component Analysis. Hindawi 2020; Article ID 3407907, pp: 8.

16. Eslami. T, S. Raiker. J, Saeed. F. Explainable and Scalable Machine-Learnig Algorithms for Detection of Autism Spectrum Disorder Using fMRI Data. Neurons and Cognition 2020; arXiv:2003.01541.

17. Thomas R.M, Gallo. S, Cerliani. L, Zhutovsky. P, El-Gazzar. A, Wingen GV. Classifying Autism Spectrum Disorder Using the Temporal Statistics of Resting-State Functional MRI Data with 3D Convolutional Neural Networks. Front. Psychiatry 2020; doi: 10.3389/fpsyt. 2020. 00440.

18. ABIDE, http: //fcon1000. p r o j e c t s. nitrcorg/i n d i / a b i d e.

19. Di Martino. A, Yan C.G, Li. Q, Denio. E, Castellanos F.X, Alaerts. K, et al. The Autism Brain Imaging Data Exchange: Towards a Large-Scale Evaluation of the Intrinsic Brain Architecture in Autism. Molecular psychiatry 2013; vol.19, no.6, pp: 1-9.

20. Poldrack R.A, Mumford J.A, Nichols T.E. Handbook of Functional MRI Data Analysis. Columbia University Libraries 2011; vol.10.1017/CBO9780511895029(1), pp: 1-1291.
21. Frackowiak. R, Ashburner. J, Penny. W, Zeki. S. Human Brain Function. The Wellcome Dept. of Imaging Neuroscience 2004; 2 Edition, ISBN: $\quad 0122648412,9780122648410$.

22. Lang E.W, Tomé A.M, Keck I.R, Górriz-Sáez J.M, Puntonet C.G. Brain Connectivity Analysis: A Short Survey. Published online 2012; 1148(7), pp: 781-7.

23. Just M.A, Cherkassky V.L, Keller T.A.K, Kana R.K, Minshew N.J. Functional and Anatomical Cortical Underconnectivity in Autism: Evidence from an fMRI Study ofanExecutiveFunction Taskand Corpus Callosum Morphometry. Cerebral Cortex 2007; pp: 951-61.

24. Hull J.V, Dokovna L.B, Jacokes Z.J, Torgerson C.M, Irimia. A, Van Horn J.D. Resting-State Functional Connectivity in Autism Spectrum Disorders: A Review. Published NCBI 2017; pp: 205.

25. Tzourio-Mazoyer. N, Landeau. B, Papathanassiou. D, Crivello. F, Etard. O, et al. Automated Anatomical Labeling of Activations in SPM Using a Macroscopic Anatomical Parcellation of the MNI MRI Single-Subject Brain. Neuroimage 2002; vol.15, No.1, pp: 273-289.

26. Poli. R, Kennedy. J, Blackwell. T. Particle swarm optimization. Springer Science and Business Media 2007; pp: 1: 33-57.

27. Cortes. C, Vapnik. V. Support Vector Network. Mach Learn 1995; vol.20, pp: 273-297.

28. Ulfarsson M.O, Solo. V. A Semiparametric PCA Approach to fMRI Data Analysis. International Conference on Acoustics Speech and Signal Processing (ICASSP) 2010; pp: 634-637. 